\title{
Annemarie Matzke, Ulf Otto \& Jens Roselt (Hrsg.) Auftritte. Strategien des In-Erscheinung-Tretens in Künsten und Medien
}

\author{
Bielefeld: transcript 2015 (= Theater, Bd. 58), 249 Seiten. \\ ISBN:978-3-8376-2392-5
}

\section{Florian Vaßen}

Die in Buchrezensionen vertretenen Ansichten und Meinungen sind die der jeweiligen Rezensentinnen und Rezensenten und reflektieren nicht notwendigerweise die Position von SCENARIO.

DerAuftrittisteiner derwichtigsten Momentein einerTheateraufführung, eine Schwelle wird dabei überschritten, und es finden entscheidende Veränderungen statt. Dieser Wechsel von Nicht-Präsenz zur Präsenz, vom Off zum On wird in dem vorliegenden Sammelband in 12 Texten zum Theater und anderen „Künsten und Medien“ unter den verschiedensten Gesichtspunkten untersucht.

Ausgehend vom „Harlekin-Prinzip“-das „Auftreten“selbst ist seine „Botschaft“ -, stellen die Herausgeber in ihrer Einleitung „K(l)eine Theorie des Auftritts“ drei Dimensionen des Auftritts als „Keimzelle von Theater“ (7) vor. Erstens: Auftritt als Ostentation „ist Ausnahmezustand und Gesellungsereignis“ und führt zur „Trennung von Handelnden und Schauenden“ (9). Zweitens: „Aus dem Auftritt geht eine Figur hervor [...]: eine Gestalt", bestimmt von „Sichtbarkeit“ und „Aufmerksamkeit“ (9f.); diese Figuration führt zu „Gemeinschaftsbildungen“, zugleich aber auch zu „Ausnahme“ und „Alleinstellung“ (9). Drittens: „Ein Auftritt erlangt seine Bedeutung erst mit seiner medialen Zirkulation." (11); dabei gibt es nicht „den Auftritt“, sondern eine große Vielfalt. Entsprechend ist eine klare Definition ebenso schwierig wie die entsprechende Theoriebildung komplex ist. „Nicht was ein Auftritt ist, ist eine sinnvolle Frage, sondern was man damit machen kann" (11). Ausgehend von diesen knappen, aber überzeugenden theoretischen Überlegungen präsentiert sich im Folgenden ein breites Spektrum an Fragen, Problemstellungen, Aspekten und Untersuchungsgegenständen.

Gabriele Brandstetter bildet mit ihrem Text „Wundertheater. Der Auftritt des Theaters" (17-32) den Ausgangspunkt: Das Theater tritt selbst auf, markiert durch „Evidenz“ und einen räumlichen und zeitlichen sowie einen Als-obRahmen, der übertreten werden kann, aber auch ganz einfach als „ein Akt des Eintretens" (25) im Sinne von enter oder entering eines „Fremden oder Anderen“ (26) erscheint.

Einen anderen Ausgangspunkt wählt Christopher Wild in seiner Analyse der „Royal Re-Entries. Zum Auftritt in der griechischen Tragödie“ (33-61), in der er, 
ausgehend von der Szene des Wartens, zunächst den „erste(n) Königsauftritt des abendländischen Theaters" (35) und die damit zusammenhängenden tragischen Ereignisse in Aischylos" „Die Perser" sowie die Dekonstruktion des „ersten Glanzauftritt(s) der abendländischen Theatergeschichte“ (44) in „Agamemnon“ desselben Autors darstellt. Dem entgegen stellt Wild die Heimkehr (nostos) von Odysseus und Orest, die verkleidet als Fremde aus fernen, gefährlichen Räumen zurückkehren und entsprechend dem göttlichen Recht der Gastfreundschaft (xenia) aufgenommen werden. Mittelpunkt dieses Auftritts ist die anagnorisis als Moment des Wechsels von Unkenntnis in Kenntnis. Der Ursprung des Auftritts findet sich in Dionysos' Ankunft bei den Theaterfestspielen.

Auf das entering und den Auftritten in der antiken griechischen Tragödie folgt in Annette Kappelers Untersuchung „Attraction universelle. Opernauftritte zwischen Ancien Régime und Aufklärung (63-83) die entrée, d.h. die erlernte Choreographie des richtigen Eintretens als Zeremonie und „Herrscherinszenierung" (64) am Hofe Ludwig XIV. und die sich daran orientierenden Theater-Auftritte. Mit der Aufklärung jedoch findet in der tragédie en musique, der Oper des Ancien Regime, einer „der elementarsten Umbrüche der europäischen Auftrittskultur“ statt, beeinflusst durch „neue naturphilosophische Diskurse“ (64). Die prunkvolle entrée, deren vertikale Struktur sich mit einem „solaren Auftritttsmodell“ (67) verbindet und so den „eintretenden Herrscher zum Mittelpunkt ihrer Bühnenfiguration“ „macht" (68), wird abgelöst durch eine neue "theatrale Bewegungsästhetik“ (69). Pantomime, légèreté und pesanteur, also „Leichtigkeit und Körperschwere“ (73), sowie Beschleunigung, Niederfallen und Schweben bestimmen die Bühne und stehen z.B. bei dem Opernreformer Christoph Willibald Gluck im Zentrum.

Nach diesen historischen Untersuchungen, die aufzeigen, wie stark die Theater-Auftritte von kulturellen Gegebenheiten abhängig sind und zugleich auf sie reagieren, wendet sich Ulf Otto in seinem Beitrag „Auftritte der Sonne. Zur Genealogie des Schweinwerfens und Stimmungsmachens" (85104) der Theatertechnik, der „Bühnenmaschinerie“ (85), zu und zeigt den großen Einfluss der Technik auf die Theaterästhetik: „am Ende einer langen Entwicklung" sind die Auftritte seit ca. 1880 „einer steigenden technischen Zurichtung ausgesetzt", was zur „Unterscheidung zwischen theatralem Auftreten und medialem Erscheinen. " (86) führt. Die Überdachung des Theaters verändert grundlegend die Situation: Das natürliche Licht ist ausgesperrt, und man kann Beleuchtung an der Decke befestigen, was dazu führt, „Licht, Raum und Publikum konsequent als zusammenhängende Teile einer konsistenten ästhetischen Produktion“"zu begreifen (92). Die Sonne ist kein „Himmelskörper“ mehr, sondern ein "Lichtschein" (93). Otto zeichnet die technische Entwicklung nach bis zu dem Wendepunkt mit Thomas Edison: Das „Glühlicht“ kommt „erstmals ohne Flamme“ aus (95) und damit gibt es keine Feuergefahr mehr, keinen Ruß, kein Flackern und weniger Hitze (vgl. 96). „Mit der Elektrifizierung der Theatersonnen hat sich anno 1895 das Verhältnis von Natur, Technik und Ästhetik im Theater grundlegend gewandelt: Das Licht beginnt zu erscheinen 
und die Sonnen werden vertrieben." (101)

Juliane Vogel kehrt in ihrer Untersuchung „Sinnliches Aufsteigen. Zur Vertikalität des Auftritts auf dem Theater" (105-119) zu der historischen Dimension zurück und zeigt, dass sich „(i)m Lauf der politischen Geschichte wie der Theatergeschichten [...] erfolgreiche Auftrittsformen zu Auftrittstopoi oder Auftrittsprotokollen“ „verfestigen“ (108). Besonders wichtig „für die Entwicklung der europäischen Auftrittskulturen [...] war vor allem das römische Triumphalprotokoll“ (109), das die actio des Auftritts als körperliches Handeln des „aufrechten Gang(s)“ und der „kontrollierte(n) Setzung der Füße“ (107) zeigt. „Kontrafaktisch“ zu diesen „jubilatorischen Auftrittsformen“ stehen „moderne Schritt- und Bewegungsformen“ mit ihrer „radikalen Informalisierung oder Depotenzierung“ (112), besonders deutlich bei dem hinkenden, stolpernden und fallenden Richter Adam in Kleists „Der zerbrochene Krug“ (vgl. 113). Die vertikale Form des aufrechten Gangs ist damit nicht mehr der alleinige Maßstab, sie wird konfrontiert mit den „nicht-souveränen Auftrittsformen“ mit ihren „durch Mangel bestimmte(n) Schrittmuster(n)“(114), was zur Folge hat, dass im heutigen Theater eine ständige Auseinandersetzung zwischen beiden Auftrittsformen zu beobachten ist.

Um das Stolpern als „Selbstentzug der Bewegung“, wie Waldenfels formuliert, geht es auch in Gerald Siegmunds Text „Die verfehlte Anrufung. Der verstolperte Auftritt in Peter Steins Torquato Tasso" (121-139). Siegmund zeigt in seiner Aufführungsanalyse Tassos dreimaliges "Stolpern im Angesicht der Macht" als „ein Mechanismus der fehlgeschlagenen Anrufung“, als Verweigerung der „normative(n) Subjektivierung“ im Sinne von „Integration von Sprache, Stimme und körperlichen Gesten“ (128f.). Gegen die „Eleganz der Bewegungen“ und gegen die "Schönheit der Goethe [U+02BB] schen Verssprache" ist das Stolpern „ein ungehöriger Vorgang“ (130), der „die schöne Ordnung“ „durcheinander bring (t)“, und steht damit zugleich für den „Einbruch der Phantasie“ (134).

Jens Roselt thematisiert in seinem Text "Phänomenologie der Rampensau“ (141-156) zunächst so wichtige Aspekte wie Lampenfieber, Schadenfreude angesichts des „Misslingens“ eines Auftritts, „Pleiten-, Pech- und PannenFormate“ (141) sowie das negativ beurteilte Phänomen der „Rampensau“ (143), um sich im Folgenden beispielhaft René Polleschs Inszenierung „Ich schau dir in die Augen, gesellschaftlicher Verblendungszusammenhang“" zuzuwenden, der sich in einem ,anderthalbstündige(n) Auftrittsversuch“ (149) manifestiert und sich gegen „Mitmachtheater" und den „Repräsentationsanspruch“ (150f.) von Theater wendet. Die „Annahme, dass sich im Theater eine Gemeinschaft konstituiert“, stellt für Pollesch eben einen „Verblendungszusammenhang dar“ (151).

Der Beitrag „Das Loch im Vorhang. Zu den Auftritten des Publikums“(157-169) von Annemarie Matzke ist insofern besonders interessant, als hier die Perspektive des Blicks zum Auftritt der Zuschauer*innen wechselt, so dass sichtbar wird, wie in der Performance „der Eintritt der Zuschauer in den Theaterraum [...] ausgestellt und als mehr oder weniger freiwilliger Auftritt inszeniert [wird], betrachtet von jenen, deren Auftritte die Zuschauer später betrachten werden“ 
(157). Dabei spielen „Raumordnungen“ und die „Konventionen der Institution Theater“ „in Abgrenzung zum Alltag" eine besondere Rolle. Es findet also eine ",doppelte Grenzziehung [U+02BB] [...] zwischen Zuschauern und Akteuren und zwischen „Theater und außertheatrale(r) Öffentlichkeit" (159f.) statt. Das Publikum hat seine Auftritte neben den von den Akteuren inszenierten auch in Zwischenrufen, Störungen, Standing Ovations und ähnlichem. Matzke unterscheidet zwischen Theaterbesuchern, Publikum und Zuschauern, eine Differenzierung, die nicht nur in Bezug auf den Auftritt von Interesse sein könnte. In einer „Theatergeschichte des Publikums“ (162) korrespondieren die verschiedenen Formen eines Auftritts mit der Theaterarchitektur, z.B. mit der Entwicklung von der barocken Theaterarchitektur zur Guckkastenbühne, bzw. mit der Raumstruktur, etwa in Performances von Abramović oder Gob Squad.

Auch Geesche Wartemann präsentiert in ihrem Text „Auftritte von Kindern. Vorführung, Inszenierung, Teilhabe“ (171-182) einen veränderten Zugang zum Aspekt Auftritt, sie fragt: Wie treten Kinder auf, wie werden sie inszeniert? „Und welche Figuren, welche Konzepte von Kindheit gehen aus diesen Auftritten hervor?" (171) Ausgehend von einer „antipädagogischen Tendenz"im aktuellen Kinder- und Jugendtheater, die Kinder werden von „,becomings“"zu „beings“, zeigt Wartemann zunächst, wie Kinder in der „Blütezeit des Kindertheaters“ im 18. und 19. Jahrhundert zwar berühmt waren, aber zugleich als fremdbestimmte und dressierte Darsteller*innen auftraten. Heute ist dagegen an die Stelle eines „defizitären Blick(s) auf Kinder" (177) eine Reflektion „der Projektionen der Erwachsenen“ (175) getreten, wie vor allem am Beispiel von Gob Squads Inszenierung „Before your very eyes“ dargestellt wird. „Die Wahrnehmungen und das Wissen der Kinder werden ernsthaft als ein alle bereichernder Beitrag zu den jeweiligen Themen und Fragestellungen gesehen" (179). Darüber hinaus wäre sicherlich interessant gewesen, in welchem Verhältnis die historischen antipädagogischen Versuche von Asja Lacis, Walter Benjamin und Bertolt Brecht zu den heutigen Experimenten stehen.

Stefan Krankenhagen entfernt sich in seiner Untersuchung „Der Aufritt der Dinge. $\mathrm{Zu}$ epistemischen und relationalen Objektbegriffen im Museum“ (183-197) vom Theater; er beschreibt, wie „anhand von Objekten in Museen, Ausstellungen und Sammlungen“ gezeigt wird, wie „Dinge ihren Zustand ändern“, wenn sie „zu historischen Objekten mit spezifischen, semantischen Qualitäten“ werden (183), d.h. zu „epistemische(n) Objekten“ mit „Erkenntnisanspruch und -gehalt" (187). Als Theaterwissenschaftler hätte mich allerdings mehr interessieren, welchen,Auftritt [U+02BB] Dinge auf der Bühne haben und welche Funktion sie damit in Inszenierungen erhalten.

In ihrem Text „Im Off. Dhorasoo und Rosencrantz und Guildenstern“ (199212) beschäftigt sich Stefanie Diekmann mit dem Off „als Ort eines Auftritts“, also der „Mise en Scène des Off“ (199), sowie mit der Teichoskopie „als ein Verfahren [...], Schauplätze, Orte, Ereignisse im Off der Bühnenhandlung existent zu setzen“ (205). Hier wird zwar „enter“ durch „exeunt" (204) ergänzt, also durch den „Gang ins Off“" (208), aber die grundlegende theatrale Bedeutung des Abgangs in Opposition zum Auftritt kommt doch nur sehr begrenzt in den 
Blick.

Bettine Menke setzt mit ihrer Darstellung „Suspensionen des Auftritts. Lulu - Pollesch" (213-244) einen wichtigen Schlusspunkt des Sammelbandes und analysiert die „Suspendierung des Auftritts“ als „physischer und symbolischer Vorgang“ (213). Die „Verweigerung" des Auftritts in Wedekinds Theaterstück verweist auf den „Raum“, in dem sich die dramatische Handlung entwickelt, dabei aber „auf ihre Grenze und damit ihr konstitutives Außen bezogen“ bleibt (216). Der „Übertritt über die Schwelle“ vom Dort nach dem „Hier" (217) basiert auf Bewegung, Unterbrechung und Anwesenheit. Menke erinnert daran, dass „das Vor- und Heraustreten eines Sprechers aus dem Chor“ (219) das antike Theater konstituiert habe, heute jedoch, etwa in Marthalers Inszenierungen, sich auflöst in „verzögerten Übergängen und multiplen Zonen des Übergangs“ sowie im „Sprechen als Zitation“ (220), was insbesondere in Polleschs Theaterarbeiten von Bedeutung ist, in denen offen bleibt, ob die Schauspieler*innen dramatischen Personen entsprechen oder ein Teil der Sprachflächen sind.

Die Autor*innen geben in ihren Untersuchungen und Analysen ein weit gefächertes Bild des ,Auftritts [U+02BB] . Aus den verschiedensten Perspektiven wird die Relevanz dieses theatralen Phänomens sichtbar, sowohl in Bezug auf die Theatergeschichte als auch für die heutige Situation. Von besonderer Bedeutung ist dabei die enge Verflechtung von Theater, Medien und kulturellgesellschaftlichem Kontext. Wünschenswert wäre es allerdings gerade bei diesem Thema gewesen, anschauliches und aussagekräftiges Bildmaterial stärker in die Analysen einzubeziehen. Überrascht hat mich auch, dass das Verhältnis von Auftritt und Abtritt/Abgang sowie Anwesenheit nur sehr am Rande, vor allem in dem Beitrag von Diekmann, in den Blick geraten ist. Gerade die spezifische Semantik von Abtritt - auch als „einfachem Abort" (Deutsches Universalwörterbuch) - bzw. die Differenz von Auf-Tritt und Ab-Gang scheint mir grundlegende Überlegungen wert. Kritisch anzumerken ist schließlich, dass, abgesehen von der kurzen Einleitung und wenigen Theorie-Passagen in einzelnen Beiträgen, keine ausführliche und differenzierte theoretische Fundierung des theatralen Phänomens Auftritts stattfindet, auch wenn sich, wie es in der Einleitung heißt, der Auftritt nicht „definieren“ lasse und sich auch nicht zur „Theoriebildung“ eigne (11). Insgesamt bietet dieser Sammelband aber für Theaterwissenschaftler*innen, -praktiker*innen und auch Theaterpädagog*innen wichtige informative und neue Einsichten und Anregungen in die Auftrittsstruktur von Aufführungen und Inszenierungen. 